\title{
The Modeling and Simulation of Spaceborne SAR Moving Vessel Imaging Based on Matlab Class
}

\author{
LAO Guochao ${ }^{1,2, a}$, LIU Guozhu ${ }^{1}$, WU Xu X $^{2, b}$ \\ ${ }^{1}$ State Key Laboratory of Complex Electromagnetic Environment Effects on Electronics and \\ Information System, Luoyang 471003, China \\ ${ }^{2}$ Equiment Academy, Beijing 101416, China \\ alaoguochao@126.com, bwuxu0819@163.com
}

Keywords: spaceborne SAR; moving vessel imaging; Matlab class; modeling and simulation

\begin{abstract}
Moving vessel imaging by spaceborne Synthetic Aperture Radar (SAR) is one of the most complex SAR systems, but its modeling and simulation is mostly procedural oriented functional programming in Matlab. Aiming to improve the convenience and efficiency, and enhance the expansibility and portability of this system, an object oriented programming method based on Matlab class is presented with modeling structure and simulation procedure. The rationality of the modular system is proved and the validities of SAR and ISAR imaging process are verified by the imagery results obtained in the simulation test.
\end{abstract}

\section{Introduction}

MATLAB, short for Matrix Laboratory, is a popular math program tool with abundant functions and powerful toolboxes, which has been widely applied in different fields such as modeling and simulation, signal processing, picture processing, engineering computing, economic analysis and so on [1]. Within dozens of updates since published in 1980s, the disadvantages of Matlab that low computing efficiency and large memory occupation are modified continually and more new functions are provided frequently, especially the support for class makes Object Oriented Programming (OOP) to be possible [2].

The Synthetic Aperture Radar (SAR) with all-weather all-time remote sensing ability is always the researching hot and key point [3]. The study of moving vessel imaging by spaceborne SAR, covering modeling and simulation of satellite orbit and posture, target movement, earth rotation, echo signal generation, SAR and ISAR imagery processing, etc., is the most complex system of various SARs [4,5]. Therefore, the modeling and simulation of the procedure plays an important role in this research.

Although $\mathrm{C}$ or $\mathrm{C}++$ language is able to achieve the purpose, its operations of management, debugging and drawing, etc. are less convenient compared with using Matlab. However, the traditional method of Matlab is Procedure Oriented Programming (POP) relying heavily on variables and functions. Aiming to improve convenience and efficiency, and enhance the expansibility and portability of the system, an OOP modeling and simulation method based on class in Matlab is presented in this paper.

\section{Object Oriented Programming of Matlab Class}

There are several advantages of OOP than POP, such as decomposing a large topic into several small issues; finishing certain task by objects composition and message passing; reusing code by inheritance; independence of one module which would not influence or be influenced by the modification or addition of another module.

The class, as the centerpiece of OOP, is the abstract of the general characters of specific similar objects. Although class is similar in $\mathrm{C}++$ and Matlab language, many researchers are familiar to $\mathrm{C}++$ 
class but not Matlab class. Therefore, the constitution, definition and calling, package structure of Matlab class is generally introduced as follows [2].

Matlab class is composed of Properties and Methods. The former contains variables with different properties, such as constant variables, dependent variables, hidden variables, etc. And the latter contains functions to perform a certain task. A brief definition of the SAR Class is as table 1.

Table 1 Matlab Class Defination

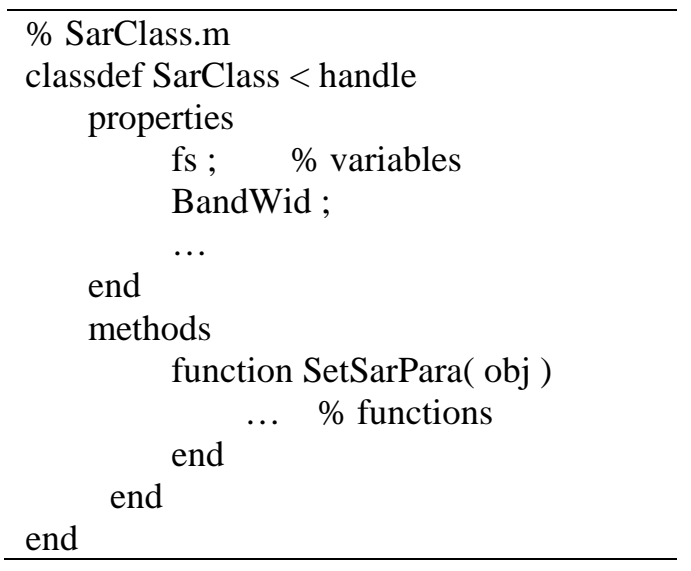

Table 2 Matlab Class Creation and Invocation

\begin{tabular}{ll}
\hline \% Simulation.m & \\
S = SarClass; & $\%$ create a class \\
S.fs = 600e6; & $\%$ evaluate a variables \\
S.SetSarPara( ); & $\%$ invoke the class \\
function & \\
\hline
\end{tabular}

Table 3 Matlab Class Package

\begin{tabular}{l}
\hline$\boxminus 13$ SarClass \\
SetBeamAngl.m \\
SarClass.m \% Class main file \\
\%
\end{tabular}

All of the relative variables and functions could be defined in a class file 'SarClass.m'. In the main simulation function 'Simulation.m', the creation and invocation of the SAR Class is as table 2. A class ' $\mathrm{S}$ ' is easily created by the equal symbol, and its variable or function is invocated by the dot symbol. All files of one class are conveniently organized as a package with head character @, as table 3.

Owe to the class package, the file structure is much more succinct and easily managed than functional Matlab codes. Moreover, class supplies a way to transfer parameters and encapsulate into a module.

\section{The Modeling and Simulation of Spaceborne SAR Moving Vessel Imaging}

Moving Vessel Imaging by spaceborne SAR is the most complex imaging process. Two kinds of movement are contained in this procedure, one is the known movement between satellite and illuminating area on the earth, the other one is uncertain caused by vessel voyaging and rotating (i.e. roll, pitch, yaw for three dimensions). The traditional SAR imaging processing could correct the firm phase change caused by former movement, meanwhile, to compensate the blurry phase error caused by the latter, the ISAR imaging method is needed essentially [5].

Consequently, the modeling and simulation system of spaceborne SAR moving vessel imaging shall have the following modules: one main function for simulation; one class containing constant variables; four classes of SAR platform and signal, moving vessel target, SAR imaging and ISAR imaging, respectively. The whole structure is as fig. 1, and the file structure is as table 4 .

Each module in the system could perform its task well independently without influence by each other, and could be ported to other applications expediently. For example, the SarImgClass could be directly adopted to processing the echo signal data from a radar receiver as an imagery tool.

The simulation procedure is as fig. 2 . 


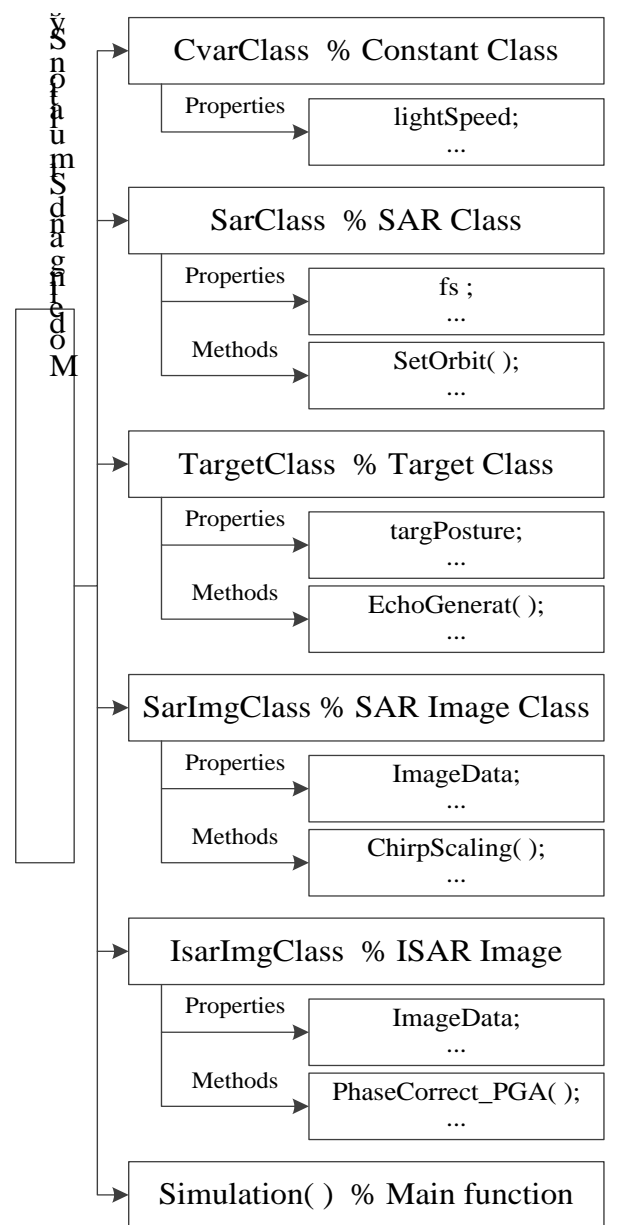

Figure 1 Modeling and Simulation System
Table 4 Simulation system file structure

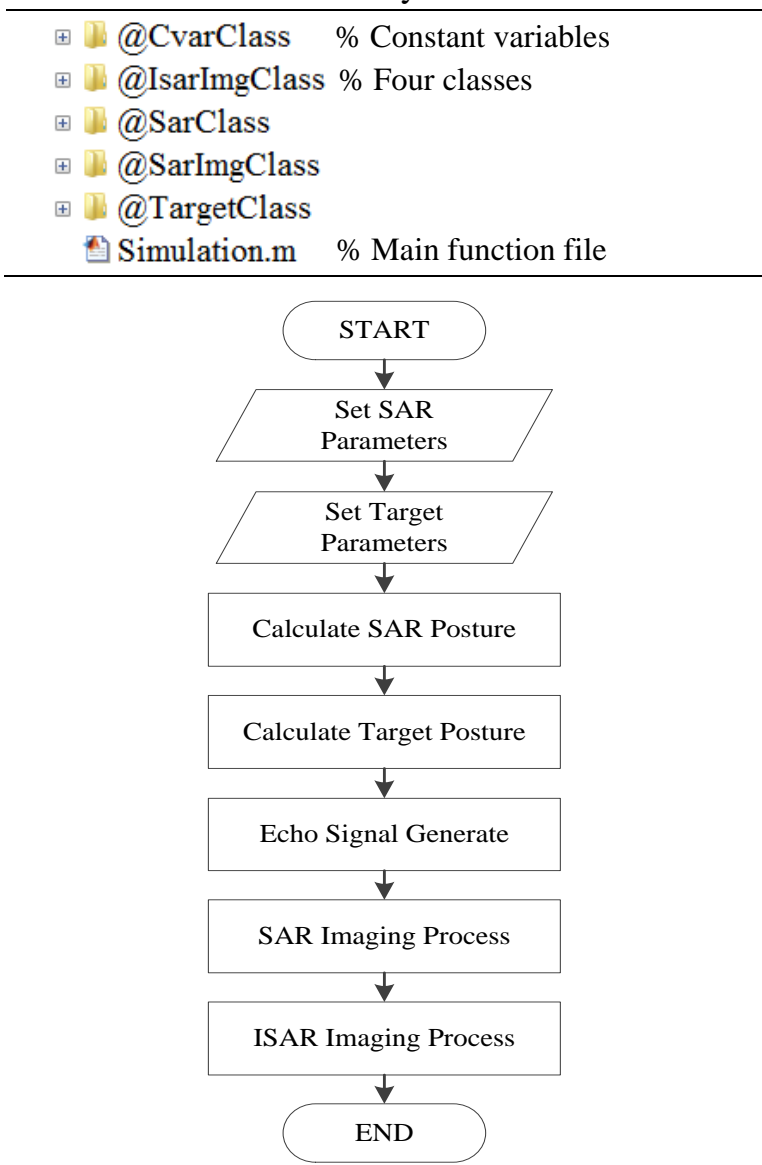

Figure 2 The simulation flowchart

\section{Simulation and Result}

Modeling and simulation system of spaceborne SAR moving vessel imaging is established, and simulation parameters is set as table 5 .

Table 5 Simulation parameters

\begin{tabular}{ccrl}
\hline Object & Item & \multicolumn{2}{c}{ Value } \\
\hline & Orbit & \multicolumn{2}{c}{ Circular } \\
SAR Platform & Height & 100 & {$[\mathrm{~km}]$} \\
& Look Angle & 45 & {$[\mathrm{deg}]$} \\
& Squint Angle & 90 & {$[\mathrm{deg}]$} \\
\hline & Band Width & 300 & {$[\mathrm{MHz}]$} \\
& PRF & 400 & {$[\mathrm{~Hz}]$} \\
SAR Signal & Azimuth Time & 3 & {$[\mathrm{~s}]$} \\
& Sample Rate & 600 & {$[\mathrm{MHz}]$} \\
\hline Vessel Voyage & Velocity & {$[10,10,0]$} & {$[\mathrm{m} / \mathrm{s}]$} \\
[x, y, z] & & & \\
\hline \multirow{2}{*}{ Vessel Rotation } & Amplitude & {$[1,1,2]$} & {$[\mathrm{deg}]$} \\
[Roll, Pitch, Yaw $]$ & Period & {$[5,4,3]$} & {$[\mathrm{s}]$} \\
& Initial Phase & {$[0,0,0]$} & {$[\mathrm{deg}]$} \\
\hline
\end{tabular}

The scattering model of the vessel is as fig. 3 . 


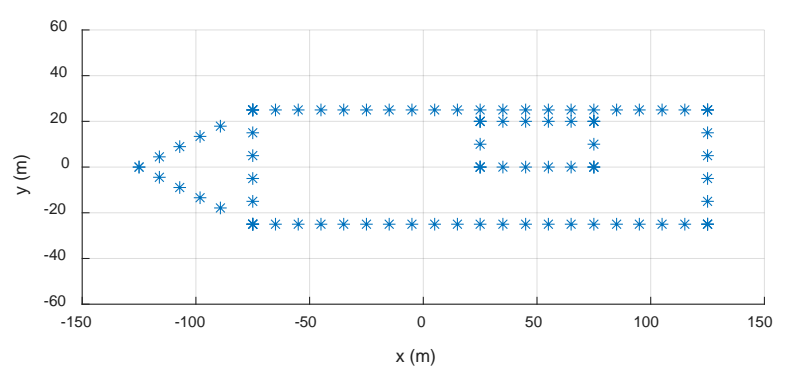

Figure 3 scattering model of vessel

The echo signal is generated at every pulse time, and then processed by SAR chirp scaling algorithm (CSA). The imaging result is as fig. 4.

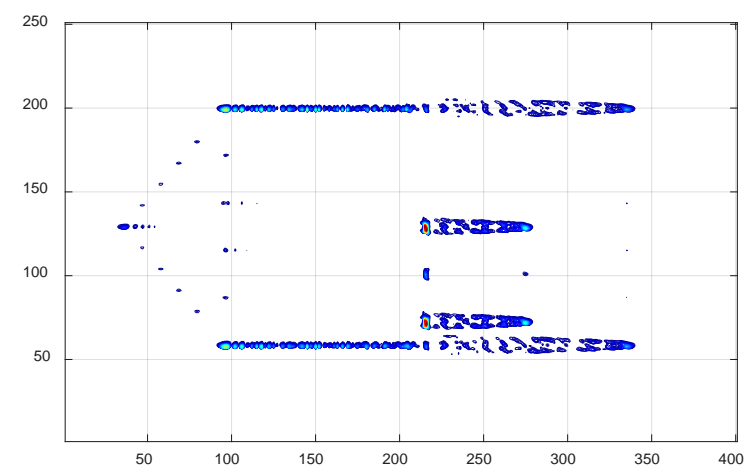

Figure 4 Imagery result by CSA (18dB)

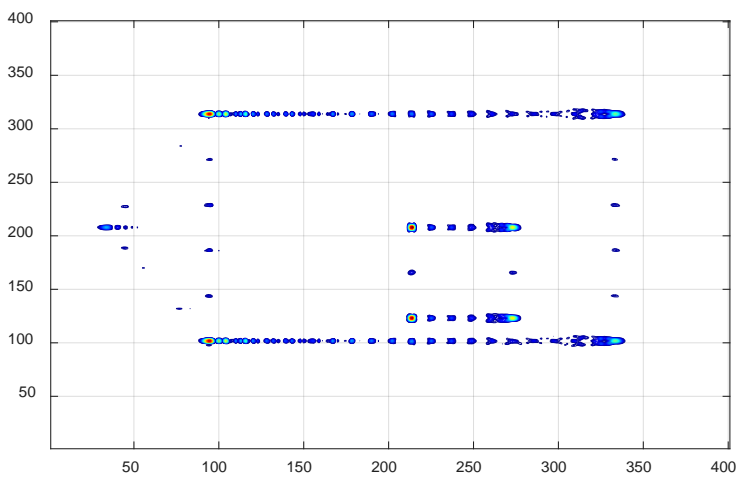

Figure 5 Imagery result by ISAR process (18dB)

The rough outline in fig. 4 is approximately coincided with the vessel model, but the scattering points are not focused well due to the phase error caused by its voyage and rotation movements. Hence, the ISAR imaging process is needed. The ISAR imaging process contains envelope aligning, phase error compensation, azimuthal imaging, etc. The ISAR imagery result is as fig. 5.

The scattering points in fig. 5 are focused better and the details are much clearer than those in fig. 4. The position of most points better seems like the vessel model.

\section{Conclusion}

On the basis of introducing the usage of Matlab class, the modeling structure and simulation procedure of spaceborne SAR moving vessel imaging are presented, whose advantages are discussed also. The results of SAR imaging algorithm and ISAR imaging process are obtained in the simulation, according to which, the rationality of the simulation is proved, and the validities of SAR algorithm and ISAR process are verified.

\section{References}

[1] Information on https://www.mathworks.com/products/matlab.html

[2] X Xv, Y Li: Object Oriented Programming in MATLAB (Beihang University Press, Beijing, 2015)

[3] I. G. Cumming, F H Wong: Digital Processing of Synthetic Aperture Radar Data Algorithms and Implementation (Artech House Print, Demand, 2005)

[4] X Xing, K Ji, LK and M Zhan: Review of Ship Surveillance Technologies Based on High-Resolution Wide-Swath Synthetic Aperture Radar Imaging. Journal of Radars. Vol. 4(2015), p. 107-121.

[5] J Gao: ISAR Ship Imaging and Crossrange Scaling with Multipath and Clutter Interference (Harbin Institute of Technology, Harbin, 2009) 